\title{
Modelos Econômicos de Complementaridade Mista
}

C. PERIN ${ }^{1}$, P. PULINO ${ }^{2}$, J.A. SCARAMUCCI ${ }^{3}$, O.F.J.G. BORDONI ${ }^{4}$, Departamento de Matemática Aplicada, Instituto de Matemática, Estatística e Computação Científica, UNICAMP, 13083-970 Campinas, SP, Brasil.

Resumo. Muitos problemas reais na área de equilíbrio energético e econômico são modelados como problemas de complementaridade mista: obter vetores $x \in \Re_{+}^{m}$, $y \in \Re^{n}$ que satisfaçam $f(x, y) \geq 0, g(x, y)=0, x^{T} f(x, y)=0$ onde $f: \Re^{m+n} \mapsto$ $\Re^{m}, g: \Re^{m+n} \mapsto \Re^{n}$ são funções conhecidas. É comum utilizar potências e logaritmos de variáveis tornando as funções de complementaridade definidas apenas para valores não-negativos destas variáveis e provocando dificuldades computacionais. Neste trabalho é feito um estudo computacional do método da transformação semisuave em exemplares disponíveis na rede mundial de computadores e em exemplares gerados a partir de dados relacionados com o equilíbrio energético do Brasil. Tais exemplares de complementaridade são gerados a partir de sua descrição pelo pacote Pegasus.

\section{Introdução}

Nos últimos anos, diversos algoritmos para problemas de complementaridade de grande porte foram desenvolvidos. Muitos têm uma apresentação teórica promissora, mas é difícil prever o seu comportamento computacional na prática. Mesmo com um conjunto de testes extensivos, é difícil comparar os métodos existentes.

Uma motivação inicial para o estudo de problemas de complementaridade linear deveu-se às condições de otimalidade de Karush-Kuhn-Tucker para programas lineares e para programas quadráticos, que constituem problemas de complementaridade linear (mista). Entretanto, o estudo destes problemas realmente cresceu de importância quando Howson e Lemke mostraram que o problema de cômputo do ponto de equilíbrio de um jogo com bimatriz pode ser posto como um problema de complementaridade linear e desenvolveram um importante procedimento com pivoteamentos. Posteriormente, Cottle introduziu o problema de complementaridade não-linear que é relacionado com o problema de desigualdade variacional.

A característica principal de um modelo de complementaridade reside no conjunto de condições de complementaridade que requer que o produto de duas ou mais quantidades não-negativas seja nulo; cada uma destas quantidades ou é uma variável

\footnotetext{
${ }^{1}$ clovis@ime.unicamp.br

${ }^{2}$ pulino@ime.unicamp.br

${ }^{3}$ scaramuc@ime.unicamp.br

${ }^{4}$ bordoni@ime.unicamp.br
} 
de decisão ou é uma função das variáveis de decisão. Tais problemas tornaram-se importantes devido ao conceito de complementaridade coincidir com a noção de equilíbrio de sistema. O balanço de oferta e demanda é fundamental para todo sistema econômico; matematicamente, esta equação é freqüentemente descrita pela relação de complementaridade entre dois conjuntos de variáveis de decisão. Por exemplo, a clássica lei Walrasiana de equilíbrio competitivo entre trocas econômicas pode ser formulada como um problema de complementaridade nos preços e nas variáveis de excesso da demanda. A complementaridade expressa o fato de que o excesso de demanda de um bem preço deve ser nulo sempre que o preço for positivo; similarmente, o preço de um bem deve ser nulo sempre que existir um excesso positivo de oferta deste bem.

O Departamento de Energia dos Estados Unidos utiliza o modelo de grande porte NEMS (National Energy Modeling System) para computar um ponto de equilíbrio de preços e quantidades de combustível para o setor de energia. Para gerar estes valores de equilíbrio, é necessário resolver iterativamente uma seqüência de programas lineares e equações não-lineares. Trata-se de uma abordagem Gauss-Seidel não-linear para obter estimativas dos valores de mercado dos preços e quantidades de combustíveis. Uma reconstrução deste modelo é proposta em [6] com argumentos convincentes. Os diversos blocos setoriais do NEMS devem ser unificados em um único bloco e constituir um modelo de complementaridade mista.

Neste trabalho estamos abordando um dos métodos mais interessantes para problemas de complementaridade, o método da transformação semi-suave, que é estudado em [9]. De modo resumido, este método utiliza uma função semi-suave para transformar o problema original de complementaridade mista em um problema de programação não-linear, que é a seguir resolvido por um método do tipo Newton. Realizamos um experimento computacional implementando este método de Newton generalizado para resolver exemplares de problemas de complementaridade mista relacionados com o equilíbrio energético do Brasil. Tais exemplares são gerados a partir de sua descrição pelo pacote Pegasus $[2,11]$. Também utilizamos exemplares disponíveis na rede mundial de computadores.

\section{Problema de Complementaridade}

Um problema de complementaridade mista (MCP) é especificado por completo com uma função $f: \Re^{n} \mapsto \Re^{n}$ e limitantes $\ell_{j} \in \Re \cup\{-\infty\}, u_{j} \in \Re \cup\{+\infty\}$. O problema consiste em determinar variáveis reais $x_{j} \in\left[\ell_{j}, u_{j}\right]$ que satisfaçam, para todo $j$,

$$
\begin{aligned}
x_{j}=\ell_{j} & \Rightarrow f_{j}(x) \geq 0 \\
\ell_{j}<x_{j}<u_{j} & \Rightarrow f_{j}(x)=0 \\
x_{j}=u_{j} & \Rightarrow f_{j}(x) \leq 0
\end{aligned}
$$

O caso especial de resolver um sistema de equações não-lineares

$$
f(x)=0
$$


é obtido quando $\ell_{j}=-\infty, u_{j}=+\infty$ para todo $j$.

Outro caso especial corresponde às condições de Karush-Kuhn-Tucker para programas não-lineares que, na forma

$$
\min \{F(y): G(y) \leq 0\},
$$

são expressas por

$$
\nabla F(y)+z \nabla G(y)=0, \quad G(y) \leq 0, \quad z \geq 0, \quad z^{T} G(y)=0,
$$

compondo um problema de complementaridade quando

$$
x=\left[\begin{array}{l}
y \\
z
\end{array}\right], \quad f(x)=\left[\begin{array}{c}
\nabla F(y)+z \nabla G(y) \\
-G(y)
\end{array}\right], \quad \ell=\left[\begin{array}{c}
-\infty \\
-\infty
\end{array}\right], \quad u=\left[\begin{array}{c}
0 \\
+\infty
\end{array}\right] .
$$

Em um modelo de equilíbrio econômico geral, as equações e relações complementares expressam basicamente as condições econômicas regulando oferta e demanda em mercados e, também, para produtores e consumidores, custo ou despesa com receita ou renda, respectivamente. As variáveis correspondentes são preços dos bens e níveis de operação de atividades e, assim, não podem assumir valores negativos. É comum empregarem-se tecnologias de produção ou preferências dos consumidores do tipo CES (elasticidade constante de substituição) (Cobb-Douglas, por exemplo). As equações e relações complementares seriam então construídas com funções não-lineares, formadas pelo produto de potências dos preços. Uma solução do problema misto de complementaridade para um modelo de equilíbrio geral corresponde a um ponto de equilíbrio walrasiano.

Já em modelos de equilíbrio econômico parcial ou setorial, os preços em alguns mercados são fixados. As relações de complementaridade são construídas freqüentemente com funções não lineares envolvendo potências ou exponenciais e variáveis não-negativas canalizadas. Uma solução do problema misto de complementaridade para um modelo de equilíbrio parcial é chamada de um equilíbrio de Cournot-Nash.

Muitos métodos têm sido empregados com sucesso para a obtenção da solução de problemas de complementaridade mista. Ferris e Pang [5] classificam as principais abordagens em: extensões do método de Newton para equações não-lineares; métodos de busca em caminhos; métodos com programação quadrática sequencial; métodos de descida baseados em otimização diferencial; métodos de projeção e proximais; técnicas suavizantes para fazer aproximações diferenciáveis de equações não-suaves; métodos de pontos interiores.

\section{Método semi-suave}

Este método trabalha com a reformulação do problema de complementaridade nãolinear mista em um sistema de equações não-lineares. Uma função $\phi: \Re^{2} \mapsto \Re$ é 
denominada NCP (de Problema de Complementaridade Não-Linear) se satisfaz

$$
\phi(u, v)=0 \quad \Leftrightarrow \quad u \geq 0, v \geq 0, u v=0
$$

como, por exemplo, $\min \{u, v\}$, mas que apresenta gradientes e subgradientes "discrepantes" em pontos relativamente próximos. Desta forma, é importante que $\phi$ também seja uma função semi-suave (vide definição em [7]) como:

- Fisher-Burmeister [9]

$$
\phi(u, v)=\sqrt{u^{2}+v^{2}}-(u+v) ;
$$

- Fisher-Burmeister penalizada [9]

$$
\phi(u, v)=\lambda\left(\sqrt{u^{2}+v^{2}}-(u+v)\right)-(1-\lambda) \max \{0, u\} \max \{0, v\}, \quad \lambda \in(0,1) ;
$$

- Kanzow-Kleinmichel [7]

$$
\phi(u, v)=\sqrt{u^{2}+v^{2}+\lambda u v}-(u+v), \quad \lambda \in(0,4) .
$$

Qualquer uma destas três funções pode ser utilizada para definir um operador $\Phi: \Re^{n} \mapsto \Re^{n}$ que satisfaça:

$$
\Phi_{j}= \begin{cases}\phi\left(x_{j}-\ell_{j}, F_{j}(x)\right), & \text { se }-\infty<\ell_{j}<u_{j}=+\infty, \\ -\phi\left(u_{j}-x_{j},-F_{j}(x)\right), & \text { se }-\infty=\ell_{j}<u_{j}<+\infty, \\ \phi\left(x_{j}-\ell_{j}, \phi\left(u_{j}-x_{j}, F_{j}(x)\right)\right), & \text { se }-\infty<\ell_{j}<u_{j}<+\infty, \\ -F_{j}(x), & \text { se }-\infty=\ell_{j}<u_{j}=+\infty .\end{cases}
$$

Note que cada solução $x$ do problema de complementaridade satisfaz $\Phi(x)=0$ e vice-versa. Além disto, $\Phi$ não é, em geral, diferenciável.

Uma abordagem importante para problemas de complementaridade mista consiste na aplicação do método de Newton não-suave (vide [10]) ao sistema $\Phi(x)=0$ globalizado com a função de mérito

$$
\Psi(x)=\frac{1}{2} \Phi(x)^{T} \Phi(x)=\frac{1}{2}\|\Phi(x)\|^{2} .
$$

Se $\Psi$ é continuamente diferenciável, defina o conjunto de $B$-subdiferenciais de $\Phi$ no ponto $x \in \Re^{n}$ como

$$
\partial_{B} \Phi(x)=\left\{H \in \Re^{n \times n} \mid \exists\left\{x^{k}\right\} \subseteq D_{\Phi}: x^{k} \rightarrow x \text { e } \Phi^{\prime}\left(x^{k}\right) \rightarrow H\right\},
$$

onde $D_{\Phi}$ denota o conjunto dos pontos onde $\Phi$ é diferenciável.

Assim o método semi-suave básico para MCP pode ser apresentado na forma abaixo (vide [9]).

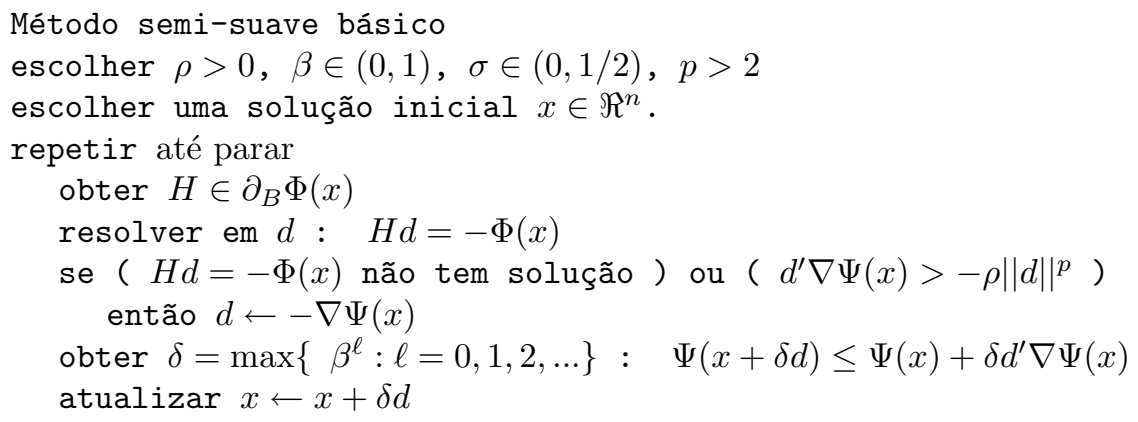




\section{Testes computacionais}

Uma grande preocupação na implementação realizada diz respeito às propriedades de convergência do algoritmo. Isto inclui aspectos numéricos relacionados com a função de mérito e o cômputo dos coeficientes do sistema linear de cada iteração, assim como o estabelecimento de critérios de parada.

Uma dificuldade na implementação do método semi-suave surge quando a função $f$ não é bem definida por não haver garantia de que os iterandos permaneçam na caixa $[\ell, u]$. Tais dificuldades surgem quando há funções de logaritmos e potências, o que é comum em muitas aplicações. Nesta implementação utilizamos, sempre que necessário, tamanhos de passo reduzidos para evitar regiões de indefinição destas funções.

\begin{tabular}{|c|c|c|}
\hline$\lambda$ & сри & $i t r$ \\
\hline 0,00 & 23 & 0,22 \\
\hline 0,10 & 73 & 0,78 \\
\hline 0,20 & 67 & 0,69 \\
\hline 0,30 & 65 & 0,70 \\
\hline 0,40 & 63 & 0,65 \\
\hline 0,50 & 62 & 0,62 \\
\hline 1,00 & 59 & 0,59 \\
\hline 1,50 & 60 & 0,63 \\
\hline 2,00 & 58 & 0,64 \\
\hline 2,50 & 57 & 0,61 \\
\hline 3,00 & 56 & 0,57 \\
\hline 3,50 & 54 & 0,61 \\
\hline 3,60 & 49 & 0,48 \\
\hline 3,70 & 51 & 0,55 \\
\hline 3,80 & 50 & 0,48 \\
\hline 3,90 & 48 & 0,52 \\
\hline 4,00 & \multicolumn{2}{|c|}{ não convergiu } \\
\hline 2,00 & 57 & 0,59 \\
\hline
\end{tabular}

Tabela 1: Número de iterações e segundos de CPU com a função de KanzowKleinmichel

Convém ressaltar que a avaliação da função de mérito é realizada com a preocupação de minimizar os erros de arredondamento. Caso $u, v$ tenham valores discrepantes, o valor computado de

$$
\phi(u, v)=\sqrt{u^{2}+v^{2}}-(u+v)
$$

pode apresentar valores diferentes dependendo da ordem em que as operações sejam 


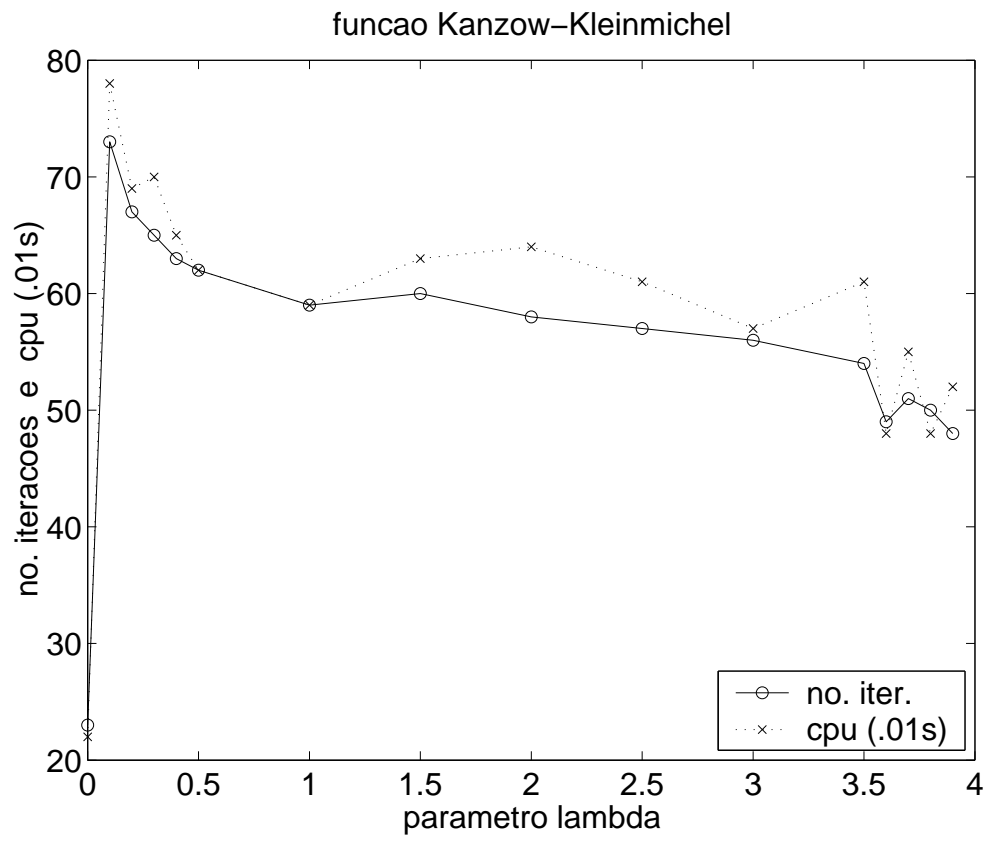

Figura 1: Número de iterações (linha contínua) e centésimos de segundos de CPU (linha pontilhada) com a função de Kanzow-Kleinmichel

realizadas. Assim, estamos efetuando esta avaliação na forma sugerida em [9]:

$$
\begin{aligned}
& \text { se }(|u|>|v|) \\
& \text { então } \phi(u, v) \leftarrow\left(\operatorname{sqrt}\left(u^{2}+v^{2}\right)-u\right)-v \\
& \text { senão } \phi(u, v) \leftarrow\left(\operatorname{sqrt}\left(v^{2}+u^{2}\right)-v\right)-u
\end{aligned}
$$

O método semi-suave foi implementado em Matlab para ambiente Windows instalado em um microcomputador pentium IV com processador INTEL $1.7 \mathrm{GHz}$ e 392MB de memória RAM. Neste trabalho estamos relatando os testes computacionais efetuados com exemplares de MCP provenientes de bibliotecas na rede internet, além de exemplares gerados a partir de problemas econômicos selecionados. Foram utilizadas as funções NCP de Fisher-Burmeister, de Fisher-Burmeister penalizada e de Kanzow-Kleinmichel.

A Tabela 1 e a Figura 1 apresentam o tempo de processamento (cpu) e o número de iterações $(i t r)$ obtidos com o uso da função NCP de Kanzow-Kleinmichel para diversos valores do parâmetro $\lambda$, em um dos problemas de equilíbrio econômico global:

$$
\phi(u, v)=\sqrt{u^{2}+v^{2}+\lambda u v}-(u+v), \quad \lambda \in(0,4) .
$$




\begin{tabular}{c|rr}
\hline$\lambda$ & \multicolumn{1}{|c}{$c p u$} & $i t r$ \\
\hline 0,00 & não & convergiu \\
0,10 & 8 & 0,08 \\
0,20 & 8 & 0,07 \\
0,30 & 8 & 0,07 \\
0,40 & 9 & 0,07 \\
0,50 & 9 & 0,07 \\
0,60 & 9 & 0,06 \\
0,70 & 8 & 0,07 \\
0,80 & 8 & 0,07 \\
0,90 & 8 & 0,07 \\
0,92 & 11 & 0,09 \\
0,94 & 144 & 1,46 \\
0,96 & 123 & 1,27 \\
0,98 & 71 & 0,73 \\
1,00 & 57 & 0,60 \\
\hline $1,00 \dagger$ & 56 & 0,59 \\
\hline
\end{tabular}

Tabela 2: Número de iterações e segundos de CPU com a função de FisherBurmeister penalizada. †função de Fisher-Burmeister

Pode-se observar que o tempo computacional e o número de iterações tendem a cair com o aumento do parâmetro $\lambda$. Entretanto, para este exemplar específico, o método não convergiu com $\lambda=4$ e o melhor desempenho foi obtido com $\lambda=0$. Infelizmente, estes dois valores específicos não garantem as propriedades de semisuavidade desta função NCP.

A Tabela 2 e a Figura 2 apresentam o tempo de processamento (cpu) e o número de iterações $(i t r)$ obtidos com o uso da função NCP de Fisher-Burmeister penalizada para diversos valores do parâmetro $\lambda$, em um dos problemas de equilíbrio econômico global:

$$
\phi(u, v)=\lambda\left(\sqrt{u^{2}+v^{2}}-(u+v)\right)-(1-\lambda)(\max \{0, u\} \max \{0, v\}), \quad \lambda \in(0,1) .
$$

Pode-se observar um comportamento indesejável para este exemplar com valores de $\lambda$ próximos de 1 . Para este exemplar, valores de $\lambda$ mais ao centro deste intervalo sugerem que o tempo computacional e o número de iterações decresçam levemente com o aumento de $\lambda$.

O valor do parâmetro $\lambda$ pode ter um impacto significativo no comportamento do algoritmo semi-suave. Pequenos valores de $\lambda$ não devem ser utilizados pois a soma de uma parcela com peso $\lambda$ pequeno com uma parcela com peso $1-\lambda$ de um termo $\max \left\{0, f_{j}(x)\right\} \max \left\{0, x_{j}\right\}$ pequeno pode contribuir para que o critério de parada seja satisfeito antecipadamente. Deve-se ressaltar que a implementação não pode utilizar aritmética exata e que o valor da função de mérito é inicialmente grande e 


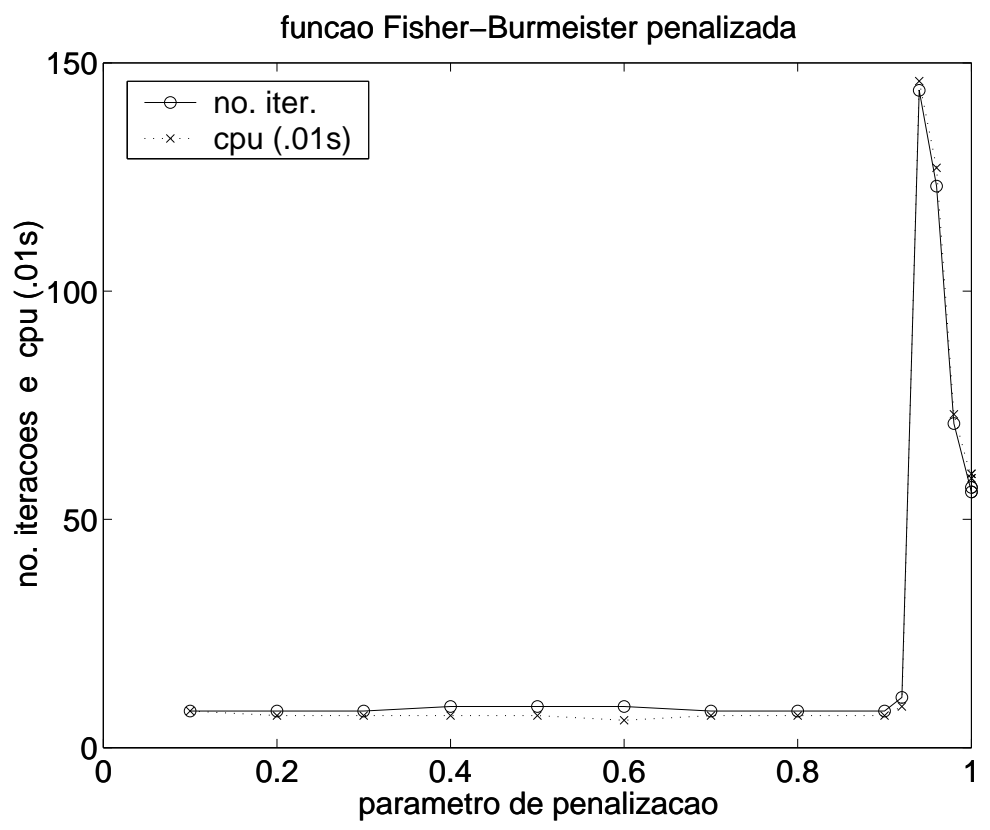

Figura 2: Número de iterações (linha contínua) e centésimos de segundos de CPU (linha pontilhada) com a função de Fisher-Burmeister penalizada

muito pequeno ao final. Ou seja, este critério pode conduzir a um ponto que satisfaz à tolerância de parada mas que não está próximo da solução, principalmente para exemplares degenerados que satisfazem $x_{j}=f_{j}(x)=0$ na solução.

A Tabela 3 apresenta o tempo de processamento (cpu) e o número de iterações (itr) obtidos para diversos exemplares testados e diferentes inicializações dos algoritmos. A coluna (par) se refere a valores predefinidos para certos parâmetros do modelo, assim como a diferentes pontos de partida predefinidos. Todos foram resolvidos com o pacote implementado com a função NCP de Fisher-Burmeister. Exceto aqueles identificados com a sigla $m c p$, os demais foram obtidos a partir das bibliotecas com exemplares em GAMS e AMPL [4]. Os problemas são fornecidos para teste e estão associados a diversos parâmetros e diversas soluções de partida. A convergência foi alcançada para todos os casos em que o ponto de partida não era um ponto de fronteira. A convergência com os pontos de fronteira foi alcançada com uma outra versão do programa, que não está apresentada neste trabalho, e que realiza um pré-processamento do sistema a ser resolvido a cada iteração. 


\begin{tabular}{l|crr}
\hline Problema & par & cpu & itr \\
\hline mcp & 1 & 8 & 0,07 \\
mcp & 2 & 12 & 0,17 \\
mcp & 3 & 57 & 0,59 \\
bertsekas & 1,1 & 25 & 0,59 \\
bertsekas & 2,1 & 22 & 0,50 \\
bertsekas & 1,2 & 23 & 0,53 \\
bertsekas & 2,2 & 19 & 0,42 \\
bertsekas & 1,3 & 25 & 0,58 \\
bertsekas & 2,3 & 19 & 0,44 \\
choi & & 6 & 1,36 \\
josephy & & 17 & 0,14 \\
kojshin & & 7 & 0,08 \\
pies & & 47 & 3,65 \\
\hline
\end{tabular}

Tabela 3: Segundos de CPU e número de iterações

\section{Considerações finais}

Os modelos estudados são de equilíbrio econômico e requerem o cálculo de funções potenciais e logarítmicas. Os resultados foram obtidos com uma versão do método de Newton generalizado, que evita a avaliação de potências e logaritmos para valores não-positivos por meio da redução do tamanho de passo. Na realidade, é utilizado um fator 0,999995 no tamanho do passo até a fronteira definida pelas variáveis nãonegativas. Trata-se de um artifício utilizado em métodos de pontos interiores para programas lineares. Uma outra versão, que não utiliza este fator de redução do tamanho de passo e que permite que a fronteira seja alcançada, apresentou, para alguns exemplares, um desempenho melhor com um número menor de iterações. Entretanto, para outros exemplares, apresentou um desempenho pior, sem alcançar a solução. De qualquer forma, não estamos apresentando os resultados obtidos com esta outra versão.

Uma versão deste método, implementada em FORTRAN 95, resolve sistemas de equações lineares esparsos com o método dos gradientes biconjugados [13] e tem apresentado um bom comportamento para sistemas de grande porte.

Abstract. Many real world problems in the area of energetic and economic equilibrium are modelled as mixed complementarity problems: find vectors $x \in \Re_{+}^{m}$, $y \in \Re^{n}$ satisfying $f(x, y) \geq 0, g(x, y)=0, x^{T} f(x, y)=0$ where $f: \Re^{m+n} \mapsto \Re^{m}$, $g: \Re^{m+n} \mapsto \Re^{n}$ are known functions. Frequently, powers and logarithms of variables are used, and the complementarity functions become defined only for nonnegative values of these variables, causing computational difficulties. In this work we perform a computational study of the semismooth transformation method applied on instances available in the world wide web as well as on instances generated from energetic sector data in Brazil. These complementarity instances are generated from their description by the Pegasus package. 


\section{Referências}

[1] S.C. Billups, S.P. Dirkse e M.C Ferris, A comparison of large scale mixed complementarity problem solvers, Computational Optimization and Applications, 7 (1997) 3-25.

[2] O.F.J.G. Bordoni, "Métodos quantitativos para construção, calibragem e solução de modelos computáveis de equíbrio geral de grande porte", Tese de Doutorado, IMECC, UNICAMP, Campinas, SP, 2001.

[3] T. De Luca, J.-S. Pang e R.E. Stone, A semismooth equation approach to the solution of nonlinear complementarity problems. Mathematical Programming 75 (1996), 407-439.

[4] S.P. Dirkse e M.C. Ferris, MCPLIB: A collection of noninear mixed complementarity problems, Optimization Methods and Software, 5 (1995), 319-345.

[5] M.C. Ferris e J.S. Pang, Engineering and economic applications of complementarity problems, SIAM Rev., 39 (1997), 669-713.

[6] S.A. Gabriel, A.S. Kydes e P. Whitman, The national energy modeling system: a large-scale energy-economic equilibrium model, Opns. Res., 49 (2001), 14-25.

[7] C. Kanzow e H. Kleinmichel, A new class of semismooth Newton-type methods for nonlinear complementarity problems, Computational Optimization and Applications, 11 (1998), 227-251.

[8] C.E. Lemke e J.T. Howson, Equilibrium points of bimatrix games, SIAM Journal of Applied Mathematics, 12 (1964), 413-423.

[9] T.S. Munson, F. Facchinei, M.C. Ferris, A. Fischer e C. Kanzow, The semismooth algorithm for large scale complementarity problems, INFORMS Journal on Computing, 13 (2001), 294-311.

[10] L. Qi J. Sun, A nonsmooth version of Newton's method, Mathematical Programming, 58 (1993), 353-367.

[11] J.A. Scaramucci, "Análise aplicada de equilíbrio econômico com Pegasus e Mathematica", Tese de Livre-Docência, IMECC, UNICAMP, Campinas, SP, 1997.

[12] D. Sun e L. Qi, On NCP-functions, Computational Optimization and Applications, (2000), 1-21.

[13] H.A. Van der Vorst, BI-CGSTAB: a faster and smoothly converging variant of BI-CG for the solution of Nonsymmetric linear systms, SIAM J. Sci. Stat. Comput., 13 (1992), 631-644. 